\title{
Ventricular Dysrhythmias after Congenital Heart Surgery: a Canine Model
}

\author{
ARTHUR GARSON, JR.
}

The Lillie Frank Abercrombie Section of Cardiology, Department of Pediatrics, Baylor College of Medicine and Texas Children's Hospital, Houston, Texas 77030

\begin{abstract}
To examine the possible mechanisms of ventricular dysrhythmias in the presence of right ventricular hypertension and following ventriculotomy, we instrumented 6-month-old puppies. There were four groups: 1) six animals served as controls (instrumentation only); 2) six animals underwent ventriculotomy only; 3) six animals underwent pulmonary artery banding with a pneumatic vessel occluder to produce right ventricular hypertension; 4) six animals had both ventriculotomy and right ventricular hypertension. Each week for 8 wk, 24-h electrocardiograms and electrophysiologic studies were performed with the animals awake and unsedated. We attempted to induce ventricular dysrhythmias with premature extrastimuli, rapid pacing, isoproterenol, and vagal stimulation. The following "chronic" data were obtained in week 8 ( $p$ value for overall analysis of variance; values are mean \pm SD): heart rate $-126 \pm 16$ beats/min (no significant difference between groups; all animals); right ventricular systolic pressure: control $26 \pm 6 \mathrm{~mm} \mathrm{Hg}$, ventriculotomy $30 \pm 3$, 1984)
AV, atrioventricular
R, right
SUP, superior
$L V$, left ventricular
$L$, left
RA, right atrial

INF, inferior
\end{abstract} right ventricular hypertension $65 \pm 5$, ventriculotomy and right ventricular hypertension $75 \pm 18(p<0.001)$; right ventricular end diastolic: control $4 \pm 1 \mathrm{~mm} \mathrm{Hg}$, ventriculotomy $4 \pm 3$, right ventricular hypertension $11 \pm 5$, ventriculotomy and right ventricular hypertension $16 \pm 7$ ( $p<0.001$ ); QRS duration: control $22 \pm 5 \mathrm{~ms}$, ventriculotomy $33 \pm 7$, right ventricular hypertension $44 \pm 6$, ventriculotomy and right ventricular hypertension $49 \pm 4$ ( $p<0.01$ ); right ventricular apex-base interval with ventricular pacing: control $20 \pm 3 \mathrm{~ms}$, ventriculotomy $34 \pm 9$, right ventricular hypertension $30 \pm 5$, ventriculotomy and right ventricular hypertension $31 \pm 6(p<0.01)$. On 24-h electrocardiogram, there were no chronic spontaneous ventricular dysrhythmias in any control or ventriculotomy animal, but were present in 5 of 6 right ventricular hypertension animals and 6 of 6 ventriculotomy and right ventricular hypertension animals. At electrophysiology study, no ventricular dysrhythmias were induced in the control or ventriculotomy animals but in the right ventricular hypertension group, 5 of 6 had accelerated ventricular escape and 2 of 6 had induced ventricular dysrhythmias; in the ventriculotomy and right ventricular hypertension group, 5 of 6 had accelerated ventricular escape and the same 5 of 6 had induced ventricular dysrhythmias including two with sustained ventricular tachycardia. In four animals, the coupling interval to the dysrhythmia was dependent upon the preceding cycle, a feature of triggered activity. In

Received November 10, 1983: accepted April 23, 1984.

Reprint requests may be addressed to Arthur Garson, Jr., M.D., Pediatric Cardiology, Texas Children's Hospital. 6621 Fannin, Houston, TX 77030.

A. G. is a recipient of National Institutes of Health Young Investigator Research Award HL24916. This work was supported in part by Grant HL07190 from the National Institutes of Health, United States Public Health Service, and by a Grant from the J. S. Abercrombie Foundation. This was presented in part at the Young Investigator Competition. American Academy of Pediatrics, Section of Cardiology. conclusion, in the dog with an otherwise normal right ventricle, ventriculotomy has no lasting dysrhythmogenic effect. Chronic right ventricle hypertrophy and dilation are associated with prolonged intraventricular conduction, spontaneous and induced dysrhythmias. Ventriculotomy may be additive in prolongation of conduction and production of dysrhythmias. The mechanism for the dysrhythmias may involve triggered activity. (Pediatr Res 18:1112-1120,

\section{Abbreviations}

In a long term follow-up of patients who had had intracardiac repair of tetralogy of Fallot, $10.3 \%$ had premature ventricular contractions found on routine electrocardiogram taken more than 1 month postoperatively, and $38 \%$ of those with premature ventricular contractions died suddenly between 3 months and 10 years postoperatively. Of the patients with premature ventricular contractions, $88 \%$ had a postoperative right ventricular systolic pressure of $60 \mathrm{~mm} \mathrm{Hg}$ or more, and all had a right ventricular end diastolic pressure of $8 \mathrm{~mm} \mathrm{Hg}$ or more. All of the patients who died suddenly had prior premature ventricular contractions, a right ventricular systolic pressure of at least 70 $\mathrm{mm} \mathrm{Hg}$, and a right ventricular end diastolic pressure of at least $10 \mathrm{~mm} \mathrm{Hg} \mathrm{(11).} \mathrm{These} \mathrm{data} \mathrm{have} \mathrm{been} \mathrm{confirmed} \mathrm{by} \mathrm{other}$ investigators, and have been extended to other patients with right ventricular outflow obstruction and interventricular communication $(7,9,10)$. On the basis of these findings, we have hypothesized a relationship between elevated right ventricular systolic pressure and end diastolic pressure, ventricular dysrhythmias, and sudden death (13). This may not be the only mechanism for late sudden death after tetralogy repair. Some patients may die from late complete AV block (37). However, two of these patients that later died suddenly had complete information regarding hemodynamics, dysrhythmia, and electrophysiology. Because of this uncertainty, an animal model was sought in which complete data could be obtained.

It was the purpose of this investigation to examine the hypothesis that hemodynamics and electrical instability are related. A model was created in which the right ventriculotomy, elevated right ventricular systolic and end diastolic pressures, could be both separated and combined, and the electrophysiologic effects studied. It was the second purpose of this investigation to inves- 
tigate the mechanism of any ventricular dysrhythmias observed in association with ventriculotomy, right ventricular hypertension, or the combination. The majority of prior work on the electrophysiologic effects of fibrosis, hypertrophy, and stretch have been on either anesthetized animals or in the tissue bath $(1,2,19,26,27,33)$. In the present model, chronically instrumented, nonsedated animals were used in an attempt to model more closely the clinical situation.

\section{MATERIALS AND METHODS}

\section{INSTRUMENTATION SURGERY}

Purebred beagles of both sexes were used. The animals were 6-8 months old $(10-13 \mathrm{~kg})$ at the time of instrumentation. The procedure was carried out on a heating pad, with rectal temperature maintained at $37^{\circ} \mathrm{C}$. A venous catheter was placed in the foreleg and anesthesia was induced with methohexital (Brevital) $7 \mathrm{mg} / \mathrm{kg}$ intravenously, and maintained with additional methohexital as necessary. Penicillin G, 150,000 units $/ \mathrm{kg}$, and kanamycin, $15 \mathrm{mg} / \mathrm{kg}$, were injected intravenously. The animal was intubated and ventilated with a Harvard small animal respirator. Blood gas analysis was performed intermittently and adjustments made in ventilation to keep the values within the normal range.

Using sterile technique, an incision was made in the left side of the neck. The left vagus nerve was isolated and a nervestimulating electrode (Medtronic model 4042) was sutured into place. The wires were tunneled subcutaneously to the scruff and the neck incision was closed.

A midline thoracotomy was then performed and the pericardium was opened. Depending upon the experimental group, either none, one, or two of the following were performed: $(a)$ transmural ventriculotomy was done by the method of Fixler and Wheeler (8) beginning in the infundibular free wall and extending caudad $6 \mathrm{~cm}$ in the right ventricular free wall, twothirds of the distance to the right ventricular apex. A continuous incision was made in stages of small incisions, each sutured closed before the next small incision, eliminating the need for cardiopulmonary bypass. (b) The main pulmonary artery was encircled with an inflatable blood vessel occluder which was designed at our institution.

For chronic monitoring, the following were implanted: (a) polyvinyl catheters (external diameter, 0.07 inch) were inserted in each of the four cardiac chambers. Nine pairs of multistranded stainless steel AWG 32 wires with a loop formed at the end were sewn to the epicardium with 6-0 synthetic nonabsorbable suture. The interelectrode distance between members of a pair was 5 $\mathrm{mm}$. Two pairs were sewn to the right atrium; six pairs were sewn to the anterior right ventricle, and one pair to the left ventricular apex. $(b)$ One electrode wire was sewn to the chest wall at each end of the thoracotomy incision. This was used as a modified surface electrocardiographic lead II.

After instrumentation, all wires and cannulae were tunneled subcutaneously and exteriorized at the scruff. The pericardium remained open. The thoracotomy incision was closed in layers, and the animals was placed in a heated cage. Hematocrit determination was performed at the end of each operaton. The level was not below $30 \%$ in any animal. Each day for the next three days, the same dose of antibiotics as was administered preoperatively, was administered intravenously. Every other day for the duration of the experiment, each catheter was aspirated and injected with $0.4 \mathrm{ml}$ of Heparin solution $(10,000 \mathrm{U} / \mathrm{ml})$ to assure patency. The Heparin remained in the tubing. Periodic determination of activated partial thromboplastin time confirmed lack of systemic heparinization.

\section{CHRONIC STIMULATION AND RECORDING PROTOCOL}

Rationale. Animals were studied at a time (6-8 months) when their age corresponded to human adolescents (35). This is the time when a large number of human sudden deaths occur (11). The animals were monitored for at least $8 \mathrm{wk}$ after the operation. This time period was chosen because: $(a)$ Bassett and Gelband
(2) have shown in cats that the hypertrophic response of the right ventricle to chronic pulmonary banding occurs by 21 days postbanding; (b) Marcus et al. (22) have shown in cats that with intermittent pulmonary artery banding, right ventricular hypertrophy occurs primarily within the Ist wk, but does not change after $4 \mathrm{wk}$; (c) Myerburg et al. (26) considered an experimental myocardial infarction "chronic" after 7 days; $(d)$ in our preliminary studies, we monitored two animals in each group for 12 weeks. There were no significant differences between weeks 8 and 12 . We, therefore, allowed $4 \mathrm{wk}$ for the changes to occur and then another $4 \mathrm{wk}$ of "chronic" observation.

Instrumentation. Once per week, the animals were taken to the electrophysiology laboratory and placed in a basket lined with blankets. The animals were awake and unsedated. After an initial period of adjustment during the first recording session, animals remained quiet in the basket. Recordings were made with an Electronics for Medicine VR12 photographic recorder at a paper speed of $100 \mathrm{~mm} / \mathrm{s}$. Bipolar epicardial electrograms were recorded from members of electrode pairs. A modified lead II was also recorded. Bipolar pacing was performed with a Bloom digital programmable stimulator using isolation amplifiers. Pulses of 2-ms duration and twice diastolic threshold were used. The pressure cannulae were attached to Statham P23db pressure transducers and monitored with the Electronics for Medicine recorder.

Electrophysiology Protocol. At the beginning of each protocol, a physical exam directed toward detection of signs of congestive heart failure was performed.

Hemodynamic indices in sinus rhythm. Heart rate was averaged over 10 beats since all animals had considerable sinus arrhythmia; also measured were left ventricular and right ventricular systolic and end diastolic pressure, right atrial and left atrial phasic and mean pressures.

Conduction times. A pacing cycle length of $240 \mathrm{~ms}$ was chosen to be more rapid than even the shortest spontaneous cycle length. During right atrial pacing at this cycle length, the high right atrium to $\mathrm{Q}$ wave interval was measured. The $\mathrm{QRS}$ duration was measured using the modified lead II in sinus rhythm. During right ventricular pacing at a cycle length of $240 \mathrm{~ms}$, the interval from the pacing spike to the superior right ventricular electrogram (right ventricular apex to base interval) was measured. Finally, during left ventricular pacing at a cycle length of 240 $\mathrm{ms}$, the interval from the left ventricular pacing spike to the right ventricular apex electrogram (left ventricle to right ventricular apex interval) was measured.

Refractory periods. During right ventricular apex pacing at a cycle length of $240 \mathrm{~ms}$, the effective refractory period of the right ventricular apex was determined by introducing single premature extrastimuli into diastole in 10 -ms decrements. This was defined as the longest ventricle to pacing spike interval which failed to capture the ventricle (14).

Induction of ventricular dysrhythmias. The following procedures were performed and the response recorded: $(a)$ rapid high right atrial pacing at a cycle length of $240 \mathrm{~ms}$ for $30 \mathrm{~s} ;(b)$ rapid ventricular pacing at a cycle length of $240 \mathrm{~ms}$ for $30 \mathrm{~s}$ from the right ventricular apex and left ventricular apex; $(c)$ introduction of single and paired ventricular extrastimuli into sinus and ventricular paced rhythm at a cycle length of $240 \mathrm{~ms}$ at both the right ventricular apex and the left ventricular apex. After the ventricular effective refractory period was reached with $S_{2}, 50$ ms were added to the $S_{1}-S_{2}$ interval and an $S_{3}$ was introduced at this same interval (e.g. effective refractory period, $130 \mathrm{~ms}$; beginning $\left.S_{1}-S_{2}, 180 \mathrm{~ms} ; S_{2}-S_{3}, 180 \mathrm{~ms}\right)$. The $S_{2}-S_{3}$ was shortened in 10 -ms decrements until $S_{3}$ failed to capture; then $S_{1}-S_{2}$ was shortened by $10 \mathrm{~ms}$. This procedure was followed until $S_{2}$ failed to capture. (d) Burst ventricular pacing at the right ventricular apex and left ventricular apex at a cycle length of $170 \mathrm{~ms}$ for 17 beats was measured; $(e)$ infusion of isoproterenol was begun at a rate of $0.05 \mu \mathrm{g} / \mathrm{kg} / \mathrm{min}$ and increased until the heart rate was approximately $200 / \mathrm{min}$. Single and paired ventricular extrastimuli were then delivered into the isoproterenol-induced sinus 
tachycardia. A single "non-bundle branch reentrant" beat was defined as a nonstimulated ventricular beat which immediately followed a stimulated ventricular beat but the nonstimulated ventricular beat had a different morphology on the surface electrocardiogram and a different activation sequence on intracardiac electrograms from the last stimulated beat (20). A "pair of non-bundle branch reentry" beats were two consecutive induced beats without an intervening sinus beat. "Nonsustained ventricular tachycardia" was defined as lasting between three beats and $15 \mathrm{~s}$. "Sustained ventricular tachycardia" lasted longer than $15 \mathrm{~s}$.

Ventricular "escape" rate following left vagal nerve stimulation. This was performed $30 \mathrm{~min}$ after the heart rate returned to control values following isoproterenol infusion. Using the method of Myerburg et al. (26), the vagus nerve was stimulated (10 times/s; stimulus strength, $10 \mathrm{~V}$; pulse duration, $0.10 \mathrm{~s}$ ) with a Grass model S4 stimulator. Stimulation was then continued until sinus arrest or complete AV block occurred, allowing unmasking of the subsidiary "escape" pacemaker. The cycle length and site of the pacemaker was then recorded.

Pharmacologic intervention. One animal with induced sustained ventricular tachycardia received verapamil $(0.15 \mathrm{mg} / \mathrm{kg}$ intravenous injection plus an infusion of $0.005 \mathrm{mg} / \mathrm{kg} / \mathrm{min}$ ) and phenytoin $(15 \mathrm{mg} / \mathrm{kg})(13,14)$.

Ambulatory Electrocardiographic Monitoring. Each week during the protocol, a 24-h electrocardiogram was obtained on each animal. The coupling interval of all ventricular dysrhythmias was measured and related to the preceding cycle length (31). If more than two consecutive premature beats were recorded, the cycle length of the first five beats was measured to determine if the cycle lengths continued to shorten ("warm up") or if they alternated ("oscillation") (18). We defined "accelerated ventricular rhythm" as three or more consecutive ventricular beats at a rate less than $150 / \mathrm{min}$. This was used to correspond with the definition of "similar to the sinus rate" by Davidson (6). Ventricular tachycardia was defined as a rate of $150 / \mathrm{min}$ or greater.

\section{EXPERIMENTAL GROUPS}

The above experiments were performed in four groups of animals each containing six animals. Group I was a control group. Instrumentation only was performed. Group II had a right ventriculotomy. Group III had chronic pulmonary artery banding. During the first 2 wk after surgery, the pneumatic main pulmonary artery occluder was progressively inflated such that a right ventricular systolic pressure between 50 and $75 \%$ of systemic was obtained. During inflation of the occluder, systemic as well as right ventricular pressure was monitored. On each day, the occluder was inflated only to a level which did not result in systemic hypotension. Group IV had both a right ventriculotomy and a chronic pulmonary artery band.

\section{PATHOLOGIC EXAMINATION}

After $8 \mathrm{wk}$, each animal was sacrificed according to the guidelines of the Baylor College of Medicine: sodium pentobarbital, $30 \mathrm{mg} / \mathrm{kg}$, followed by potassium chloride, $10 \mathrm{mEq} / \mathrm{kg}$. The heart was removed, cleaned of fibrous tissue and weighed. The ventricular portion was cross-sectioned into four pieces from apex to base parallel to the AV groove. The right ventricular and left ventricular free wall thickness were measured in the midventricle (third section from the apex). The right and left ventricular cavity dimensions were measured at midventricle. Then the right and left ventricular free walls were separated from the septum and each free wall piece was weighed. To allow for differing cavity dimensions and wall thicknesses between systole and diastole, the ratio of the right ventricle to left ventricle was used.

\section{STATISTICAL METHODS}

Data for two time periods were examined for each animal: "acute" (using the 2 -wk postoperative data) and "chronic" (using the 8 -wk postoperative data. For the acute data, all four groups were first compared with the one-way analysis of variance (34). If the overall analysis of variance revealed a statistical significance among the groups $(p<0.05)$, then individual $t$ tests were performed comparing each experimental group with control, or with each other. Bonferroni's modification was applied to the critical value of the $t$ test which resulted in statistical significance (34). The $p$ values in the text are the results of individual $t$ tests. A similar analysis was performed on the chronic data. Change from acute to chronic was assessed by taking the arithmetic difference between acute and chronic for each animal and applying analysis of variance followed by the $t$ tests. Categorical difference (i.e. presence or absence of dysrhythmia) was performed by $\chi^{2}$.

\section{RESULTS}

\section{MORBIDITY AND MORTALITY}

The operative mortality (within 1 wk of surgery) was: control, $22 \%$ ( 2 of 9 ); ventriculotomy, $25 \%$ ( 3 of 12 ), right ventricular hypertension, $44 \%$ (7 of 16); and ventriculotomy and right ventricular hypertension, $50 \%$ (10 of 20). There was a similar trend in late death: control, $14 \%$ ( 1 of 7$)$; ventriculotomy, $33 \%$ (3 of 9 ); right ventricular hypertension, $33 \%$ (3 of 9), ventriculotomy and right ventricular hypertension, $40 \%$ (4 of 10). The control animals either died during a viral infection, from rupture of the pulmonary artery, or from congestive heart failure. All of these deaths were unwitnessed, two animals being found dead in the cage. Therefore it is difficult to tell if any of these were "sudden death." None of these animals was wearing a Holter at the time of death. Data from animals which later died were not included. At the end of $8 \mathrm{wk}$, all animals had at least one pair of atrial wires functioning and three pairs of ventricular wires. Over the $8 \mathrm{wk}$, the control and ventriculotomy animals showed a tendency for weight gain which was not statistically significant $(9.6 \pm 1.1$ (SD) to $10.1 \pm 2.0)$. In the other two groups, there was a tendency to weight loss which was not statistically significant $(10.4 \pm 1.1 \mathrm{~kg}$ to $10.1 \pm 1.9)$. None of the experimental animals included in the study had overt congestive heart failure.

\section{HEMODYNAMIC DATA}

Heart Rate. The heart rates of the animals did not differ significantly among the groups or between the acute and chronic period. The heart rate of all animals (mean \pm SD) in the chronic period was $126 \pm 16$ beats/min. The range was 97 to 166 .

Ventricular Pressures. In the acute period, the right ventricular systolic pressure for control animals ranged from $22-30 \mathrm{~mm} \mathrm{Hg}$ (mean 27.5). This did not differ significantly from the ventriculotomy animals. In the right ventricular hypertension and ventriculotomy and right ventricular hypertension groups, the right ventricular systolic pressure was significantly increased. In the chronic period, the control and ventriculotomy animals remained the same, while the right ventricular systolic pressure in the other two groups was further elevated. There was no significant difference in chronic right ventricular systolic pressure comparing the right ventricular hypertension animals with the ventriculotomy and right ventricular hypertension animals. The chronic right ventricular systolic pressure in three other groups averaged $70.0 \mathrm{~mm} \mathrm{Hg}$. This is compared to the chronic control right ventricular systolic pressure mean of 26.1.

The left ventricular systolic pressure in control animals was $115 \pm 15 \mathrm{~mm} \mathrm{Hg}$ in the acute period and $105 \pm 18$ in the chronic period.

The right ventricular end diastolic pressure paralleled the right ventricular systolic pressure.

\section{ELECTROPHYSIOLOGIC DATA}

$R V$ Apex to Base Interval. In the acute period all experimental groups were prolonged compared to control and, additionally, the ventriculotomy and right ventricular hypertension group was 
prolonged above the others. In the chronic period, the ventriculotomy and right ventricular hypertension group decreased significantly ( $p<0.025$ compared to the acute period) to equal the other two experimental groups; all were different from control.

QRS Duration. In both the acute and chronic period, the QRS duration was significantly prolonged in each of the experimental groups compared to control. Furthermore, in comparing the right ventricular hypertension and the ventriculotomy and right ventricular hypertension groups to the ventriculotomy group, the groups with right ventricular hypertension had a significantly more prolonged $\mathrm{QRS}$ duration. The chronic values (mean $\pm \mathrm{SD}$ ) were: control, $21.8 \pm 4.7 \mathrm{~ms}$; ventriculotomy, $32.5 \pm 6.9(p<$ 0.025 with control); right ventricular hypertension, $44.2 \pm 6.1$ $(p<0.001$ with control); ventriculotomy and right ventricular hypertension, $49.3 \pm 3.7$ ( $p<0.001$ with control).

The longest QRS in control animals was $30 \mathrm{~ms}$. Using the mean plus two standard deviations in control animals as the normal range, the upper limit of normal was $31 \mathrm{~ms}$. We therefore assumed $35 \mathrm{~ms}$ or longer as an abnormally prolonged QRS. In the acute period, this was found in 3 of 6 ventriculotomy animals, 5 of 6 right ventricular hypertension, and 6 of 6 ventriculotomy and right ventricular hypertension. In the chronic period, the only change was that all six of the right ventricular hypertension animals developed a prolonged QRS complex. Both diffusely widened QRS complexes and notched QRS complexes were found throughout the groups other than control.

\section{SPONTANEOUS DYSRHYTHMIAS ON 24-HOUR ELECTROCARDIOGRAM}

In the acute and chronic period, neither control nor ventriculotomy animals had any ventricular dysrhythmias on 24-h electrocardiogram. In the acute period, only the ventriculotomy and right ventricular hypertension group had ventricular dysrhythmias: two dogs had uniform ventricular bigeminy. In the chronic period, ventricular dysrhythmias were found in 5 of 6 right ventricular hypertension animals (two uniform premature ventricular contractions and three accelerated ventricular rhythm) and 5 of 6 in the ventriculotomy and right ventricular hypertension group (two with uniform premature ventricular contractions, one with accelerated ventricular rhythm and two with both accelerated ventricular rhythm and ventricular tachycardia).

Characteristics of Chronic Spontaneous Dysrhythmias. In the chronic period, four dogs had single or paired uniform premature ventricular contractions (Table 1). In 2 of 4 , the premature ventricular contractions were fixed coupled and in 2 of 4 (one right ventricular hypertension and one ventriculotomy and right ventricular hypertension), there was a direct relationship between the preceding cycle length and the coupling interval (Fig. 1). Five dogs had accelerated ventricular rhythm at a rate of 97-150 beats $/ \mathrm{min}$. The cycle lengths progressively shortened over the first five beats (Fig. 2). In all five of these dogs, the accelerated ventricular rhythm was sustained for long periods of time and was present for several hours per day. In one dog with sinus pauses and single accelerated ventricular escape beats, the electrocardiographic morphology of the accelerated ventricular escape beats was identical to the accelerated ventricular rhythm, which began prematurely.

Table 1. Chronic dysrhythmias on 24-h electrocardiogram*

\begin{tabular}{lcccc}
\hline & Control & Ventriculotomy & RVH & V + RHV \\
\hline PVC & 0 & 0 & 2 & 2 \\
AVR & 0 & 0 & 3 & 1 \\
VT & 0 & 0 & 0 & 2 \\
Total & 0 & 0 & 5 & 5 \\
\hline
\end{tabular}

*AVR, accelerated ventricular rhythm; PVC, premature ventricular contraction; RVH, right ventricular hypertension group; $\mathrm{V}$, ventriculotomy group; VT, ventricular tachycardia.
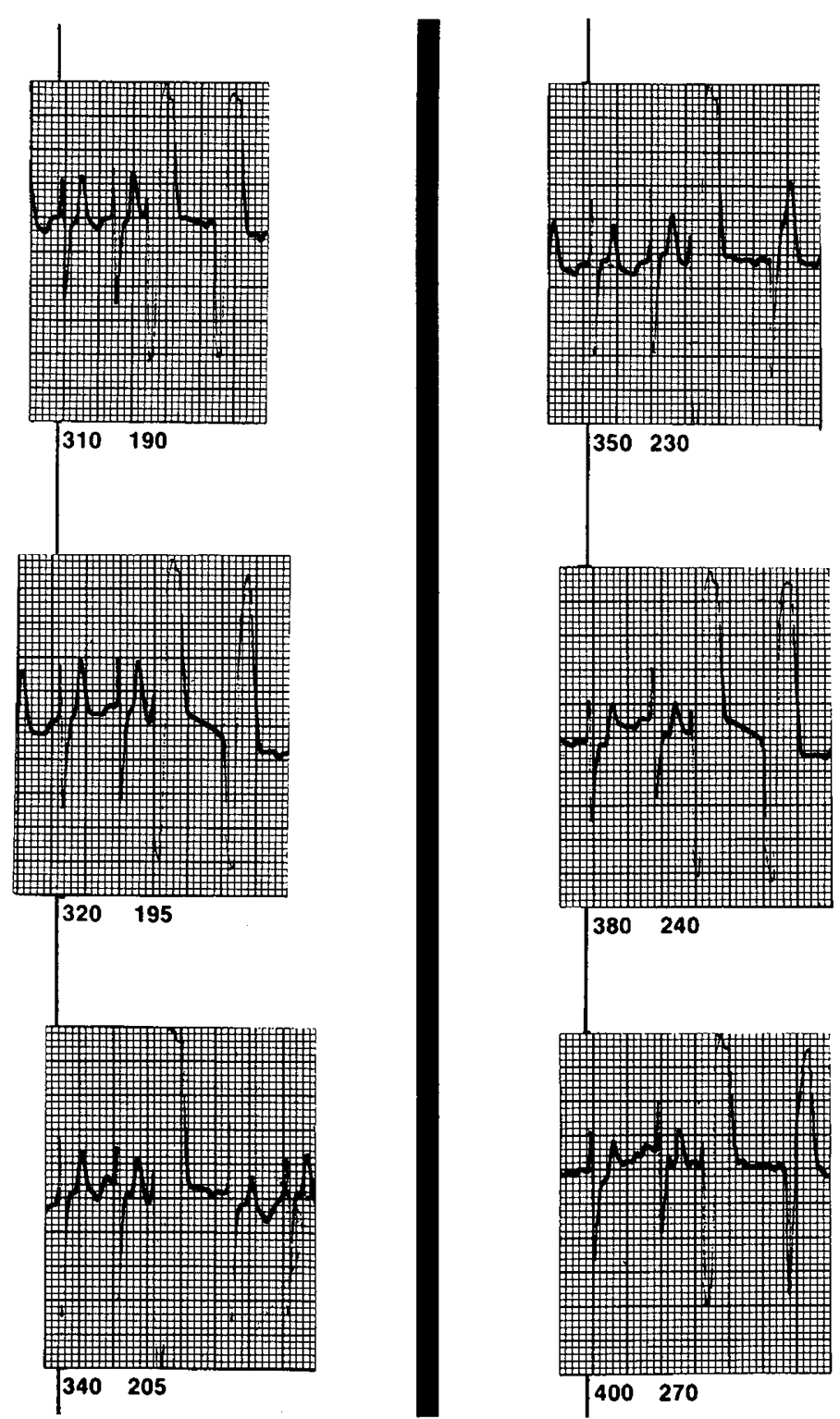

Fig. 1. Spontaneous premature ventricular contractions on 24-h electrocardiogram from a single animal. As the sinus cycle length immediately before the premature beat increases from 310 to $400 \mathrm{~ms}$, the coupling interval increases from 190 to $270 \mathrm{~ms}$.

Spontaneous ventricular tachycardia occurred in two dogs both in the ventriculotomy and right ventricular hypertension group at rates of 200 to 260 beats/min. The episodes were nonsustained and had progressive shortening of the cycle lengths.

There was no specific relationship of spontaneous or induced dysrhythmias to effective refractory period of the right ventricle, QRS duration, interventricular conduction time, or intraventricular conduction time.

Induced Ventricular Dysrhythmias. In the acute and chronic period, neither control nor ventriculotomy animals had any induced ventricular dysrhythmias (Table 2). In the chronic period, two right ventricular hypertension animals had single and paired non-bundle branch reentry beats induced. Of the six ventriculotomy and right ventricular hypertension animals, five had induced ventricular dysrhythmias in the chronic period: one had pairs, two had accelerated ventricular rhythm, and two had sustained ventricular tachycardia.

In the chronic period, of the seven dogs with dysrhythmias induced, the minimal inducing stimulus was single premature beats into ventricular paced rhythm in two dogs, double premature beats into ventricular paced rhythm in three dogs, and burst 


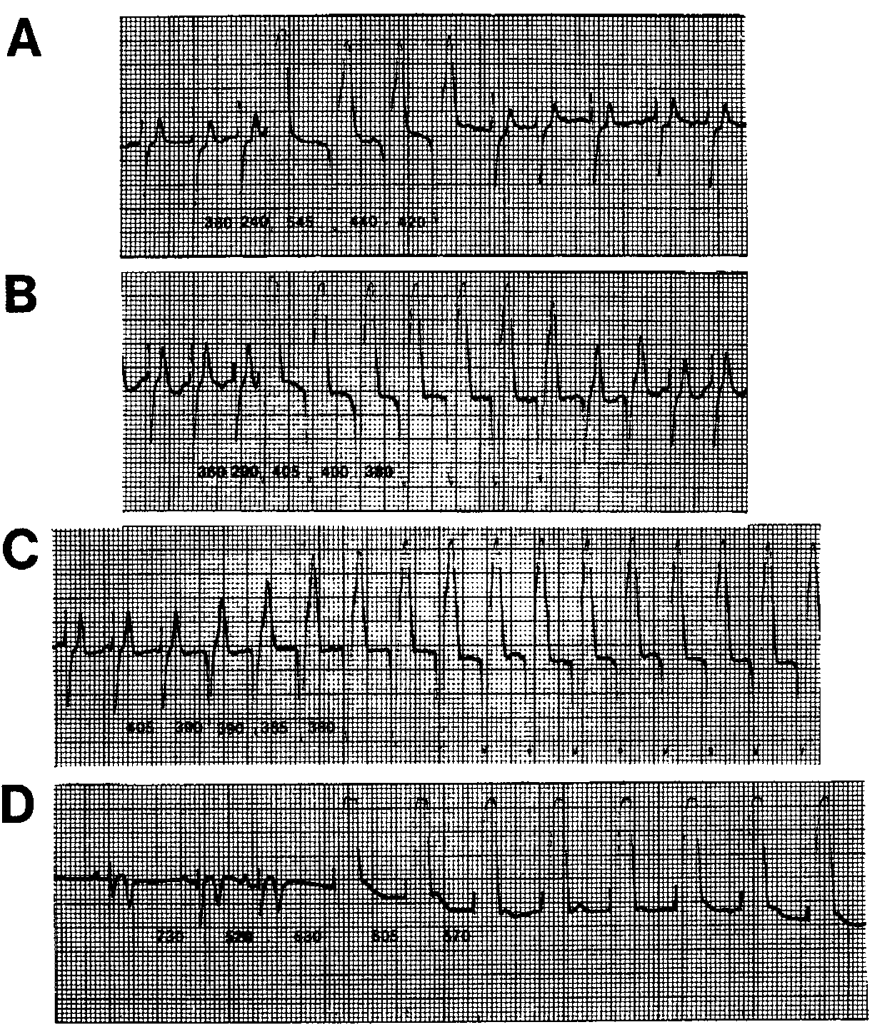

Fig. 2. Accelerated ventricular rhythm on 24-h electrocardiogram. Note the relationship to initiating cycle length similar to Figure 1. Tracings $A-C$ were taken from the same animal. Tracing $D$ was from another animal.

Table 2. Ventricular dysrhythmias induced in chronic electrophysiology study*

\begin{tabular}{lcccc}
\hline & Control & Ventriculotomy & RVH & V + RVH \\
\hline Single NBBR & 0 & 0 & 0 & 2 \\
Pair NBBR & 0 & 0 & 2 & 1 \\
Non-Sus VT & 0 & 0 & 0 & 0 \\
Sustained VT & 0 & 0 & 0 & 2 \\
Total & 0 & 0 & 2 & 5
\end{tabular}

* NBBR, non-bundle branch reentry beat; Non-Sus, nonsustained; $\mathrm{VT}$, ventricular tachycardia; others are as in Table 1.

pacing in two. These dysrhythmias were induced reproducibly over several minutes.

With the addition of isoproterenol, spontaneous single premature ventricular contractions and couplets were observed in three dogs (two ventriculotomy and right ventricular hypertension, one right ventricular hypertension) all with previously induced ventricular dysrhythmias. In one dog in the right ventricular hypertension group without previously induced ventricular dysrhythmias, in response to isoproterenol spontaneous accelerated ventricular rhythm developed with the same morphology and activation sequence as the accelerated ventricular escape beat in response to vagal stimulation. Of the seven dogs with induced ventricular dysrhythmias before isoproterenol, five had a similar dysrhythmia induced during the isoproterenol infusion. No dog had induced dysrhythmias during isoproterenol that was not found before the drug was administered.

Characteristics of Induced Dysrhythmias. Single or paired nonbundle branch reentry beats occurred in five animals. In 2 of 5 , the non-bundle branch reentry beats occurred early in diastole following the last stimulated beat (Fig. 3). These were only present at two coupling intervals and so the relationship of the coupling interval to the preceding cycle could not be determined. In 3 of 5 , the non-bundle branch reentry beats occurred late in diastole following the last stimulated beat. These were present at multiple coupling intervals and in all three, there was a direct relationship of the inducing cycle length to the coupling interval of the first non-bundle branch reentry beat (Fig. 4).

Accelerated ventricular rhythm (rate $<150$ beats/min) lasting four to five beats immediately followed introduction of premature beats into paced rhythm in two dogs (both in the ventriculotomy and right ventricular hypertension group). Whether this rhythm was actually "induced" by the pacing or whether the pacing suppressed the sinus node and allowed for the emergency of this rhythm could not be differentiated. Cycle lengths of this rhythm progressively shortened (i.e. "warmed up"). In both dogs, this rhythm had the same morphology as the ventricular escape rhythm produced during vagal stimulation (Fig. 5).

Sustained ventricular tachycardia was induced in two dogs, both in the ventriculotomy and right ventricular hypertension group. In one, there was no cycle length dependency of the tachycardia on the prematurity interval of the stimulating beat. The tachycardia stopped immediately with overdrive pacing. No drugs were given. In the other animal, there was a direct relationship of the inducing cycle length to the coupling interval of the first beat of the tachycardia and to the tachycardia cycle length (i.e. with a shorter $S_{1}-S_{2}$ interval, the interval from $S_{2}$ to the first beat of tachycardia was shorter and also, the cycle length of the succeeding beats of tachycardia were shorter) (Fig. 6). The tachycardia stopped immediately with overdrive pacing and could be reinduced with pacing or it also began spontaneously. In response to verapamil $(0.15 \mathrm{mg} / \mathrm{kg}$ intravenous injection plus an infusion of $0.005 \mathrm{mg} / \mathrm{kg} / \mathrm{min}$ ), the tachycardia cycle length lengthened and had spontaneous termination (Fig. 7). Thirty minutes after the verapamil was discontinued, sustained ventricular tachycardia returned with the same cycle length. Then phenytoin, $15 \mathrm{mg} / \mathrm{kg}$, was given intravenously over $1 \cdot \mathrm{h}(13,14)$. The tachycardia stopped spontaneously and could not be rei-nitiated.

In the animals with induced ventricular dysrhythmias, the earliest epicardial electrocardiogram was always in the right ventricle but there was no consistent specific location. In these wide-QRS beats, the right ventricular epicardial sites were all activated before, or within $40 \mathrm{~ms}$ of the onset of the QRS (Fig. 7).

Escape Site and Escape Cycle Length. In the chronic period, in control animals, all escapes occurred from the sinus or junction and the cycle length of each was greater than $1000 \mathrm{~ms}$ (Fig. 8). "Normal" was therefore defined as an escape from sinus or junction at a cycle length of $1000 \mathrm{~ms}$ or greater. Ventriculotomy animals all had a normal chronic response to vagal stimulation. On the other hand, 4 of 6 right ventricular hypertension animals and 5 of 6 ventriculotomy and right ventricular hypertension animals had an escape cycle length less than $1000 \mathrm{~ms}$ with a right ventricular origin. These nine animals, therefore, had an accelerated ventricular escape rate. In three of these animals, the ventricular escape beat was followed by 3-5 more beats with the same site of origin: an "accelerated ventricular escape rhythm." In each of these animals, during this rhythm, the cycle lengths progressively shortened (Fig. 7).

\section{PATHOLOGY}

The ratio of the right ventricular/left ventricular free wall thickness in control animals was $0.38 \pm 0.07$. Ventriculotomy animals were similar. Relative to the left ventricular free wall, the right ventricular free wall was approximately twice as thick as control in the right ventricular hypertension and ventriculotomy and right ventricular hypertension animals $(0.85 \pm 0.11$ and $0.91 \pm 0.15$; both $p<0.001$ compared to control). The ratio of the right ventricular/left ventricular cavity size in control animals was $0.49 \pm 0.14$. The ventriculotomy animals were not significantly different. Both the right ventricular hypertension and ventriculotomy and right ventricular hypertension animals had dilated right ventricles compared to control. The right ventricular/left ventricular cavity ratios for these two groups were 


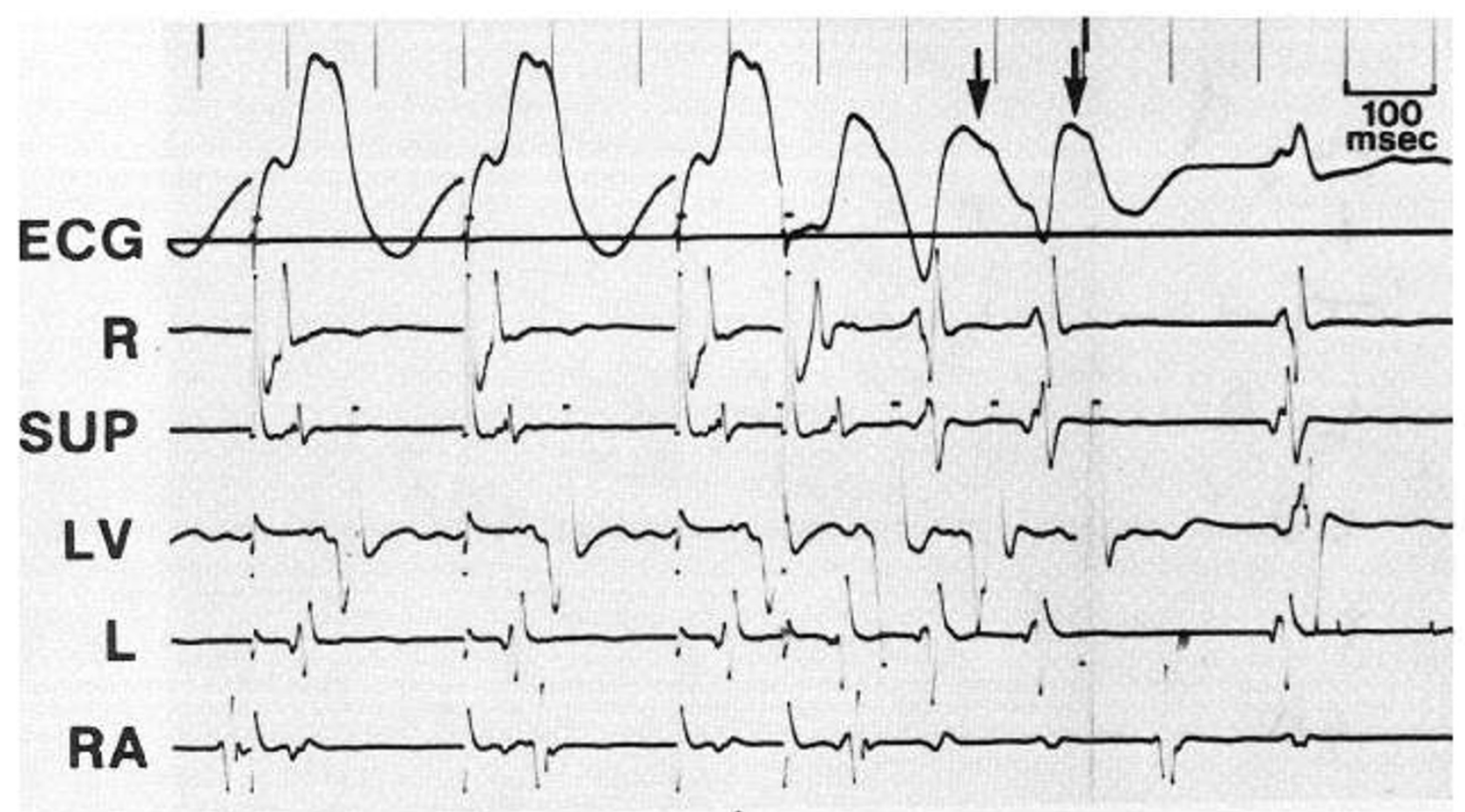

Fig. 3. Induction of a pair of non-bundle branch reentry beats (arrows) occurring early in diastole, in a dog in the right ventricular hypertension group. In the induced beats, note the earliest epicardial activation occurs in the electrode to the left of the right ventricular free wall ( $L$, electrode). It then spreads to the other right ventricular sites within $15 \mathrm{~ms}$ and to the left ventricle after $45 \mathrm{~ms}$. Note also the notched QRS in the last beat which is of supraventricular origin.

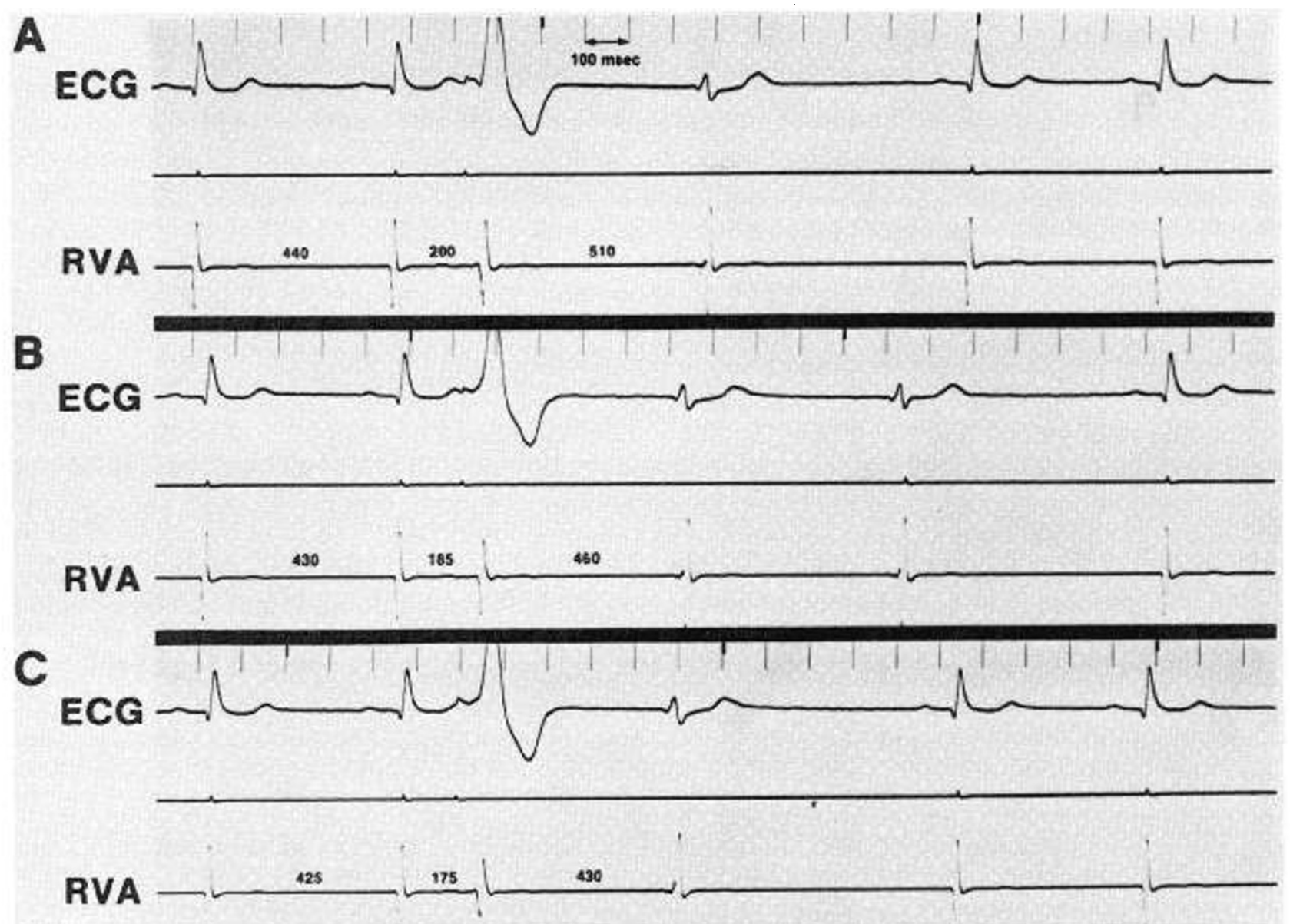

Fig. 4. Induction of single and paired non-bundle branch reentry beats occurring late in diastole. In each panel, the last two sinus beats are shown, followed by a premature ventricular extrastimulus. After the premature ventricular depolarization, there is an induced ventricular beat. As the prematurity interval to the premature extrastimulus is reduced from 200 to $175 \mathrm{~ms}$, the coupling interval to the induced beat also shortens from 510 to $430 \mathrm{~ms}$. 
A
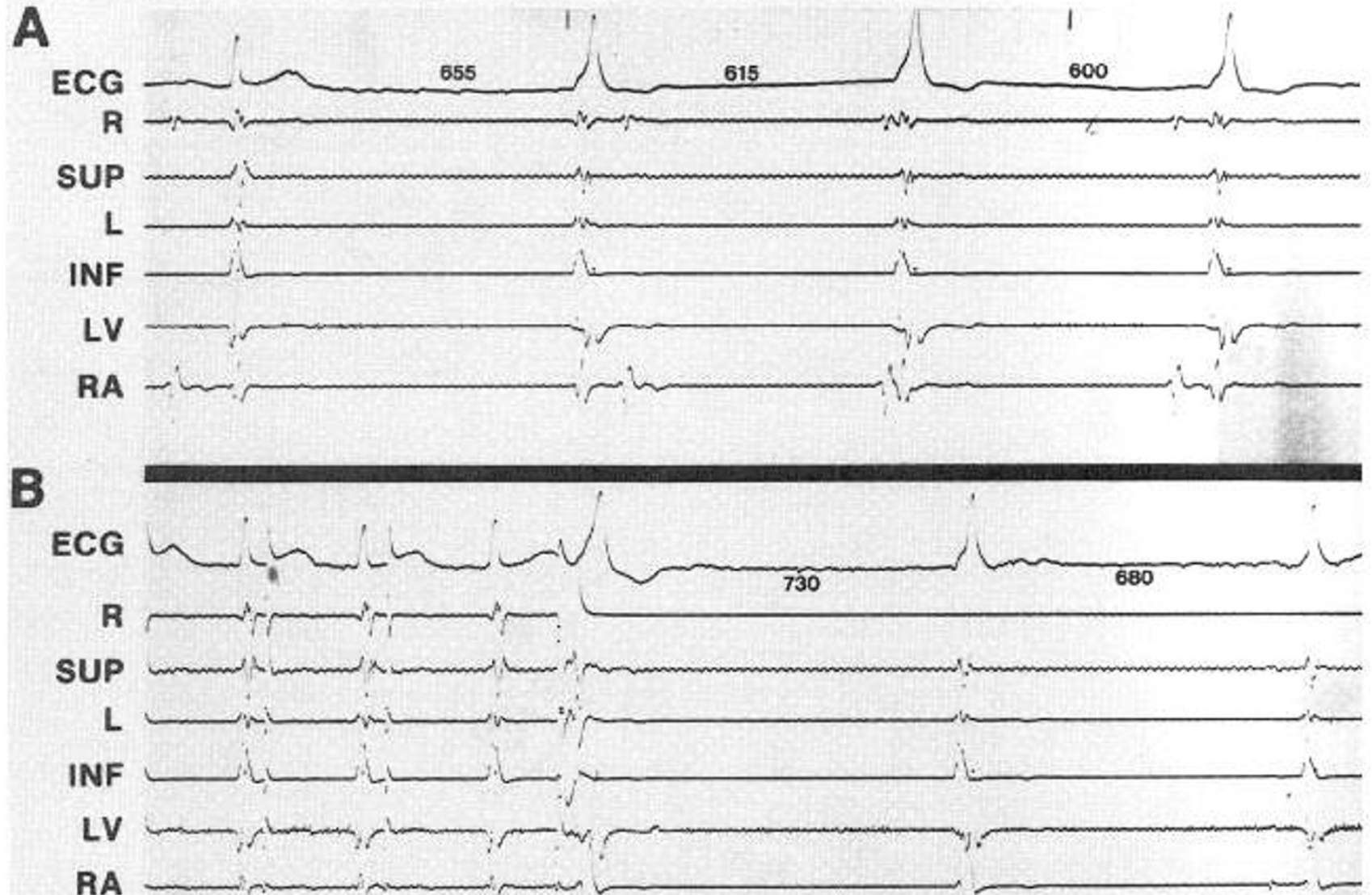

Fig. 5. Top, accelerated ventricular rhythm occurring spontaneously with vagal stimulation. Note the earliest epicardial activation in the "inferior" lead. Bottom, the last three beats of atrial pacing are shown, followed by a premature ventricular extrastimulus. After the premature ventricular depolarization, there is induced a similar rhythm with the same activation sequence as that which occurred during vagal stimulation.

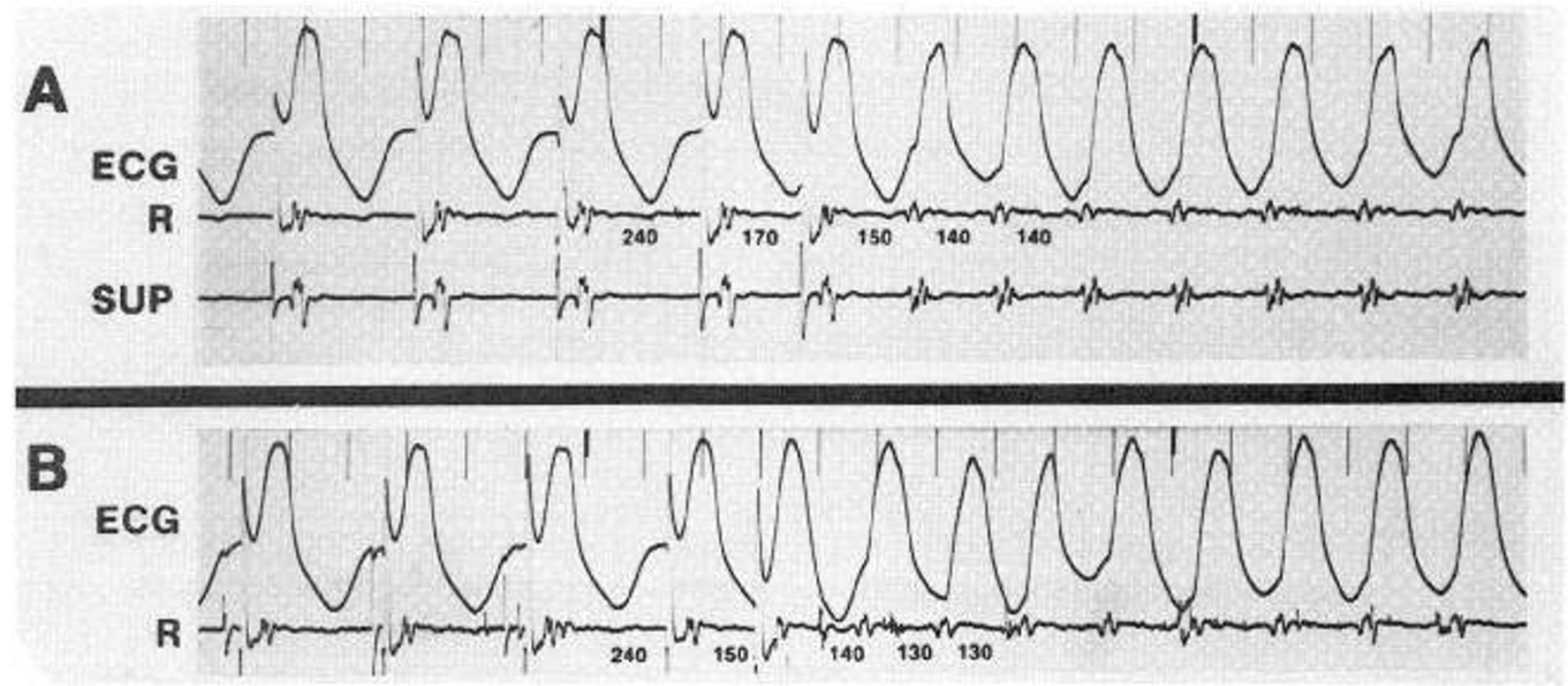

Fig. 6. Induction of sustained ventricular tachycardia. Shown are the last four ventricular paced beats followed by a premature extrastimulus. In $A$, with a prematurity interval of $170 \mathrm{~ms}$, there is a longer coupling interval and a slower tachycardia rate than in $B$ which was induced with a shorter prematurity interval.

$1.03 \pm 0.19$ and $1.17 \pm 0.21$ (both $p<0.001$ compared to control).

\section{DISCUSSION}

\section{HEMODYNAMICS AND ELECTROPHYSIOLOGY}

Ventriculotomy Group. The animals in this group had no spontaneous or induced ventricular dysrhythmias, thereby im- plying that chronic right ventriculotomy seems to be an insufficient stimulus for development of ventricular dysrhythmias. This group, therefore, has a certain similarity to children who have undergone transventricular closure of a ventricular septal defect $(17,21)$.

Right Ventricular Hypertension Group. The hemodynamics of this group were of severe right ventricular pressure overload. The right ventricular systolic pressure was greater than $50 \%$ of sys- 


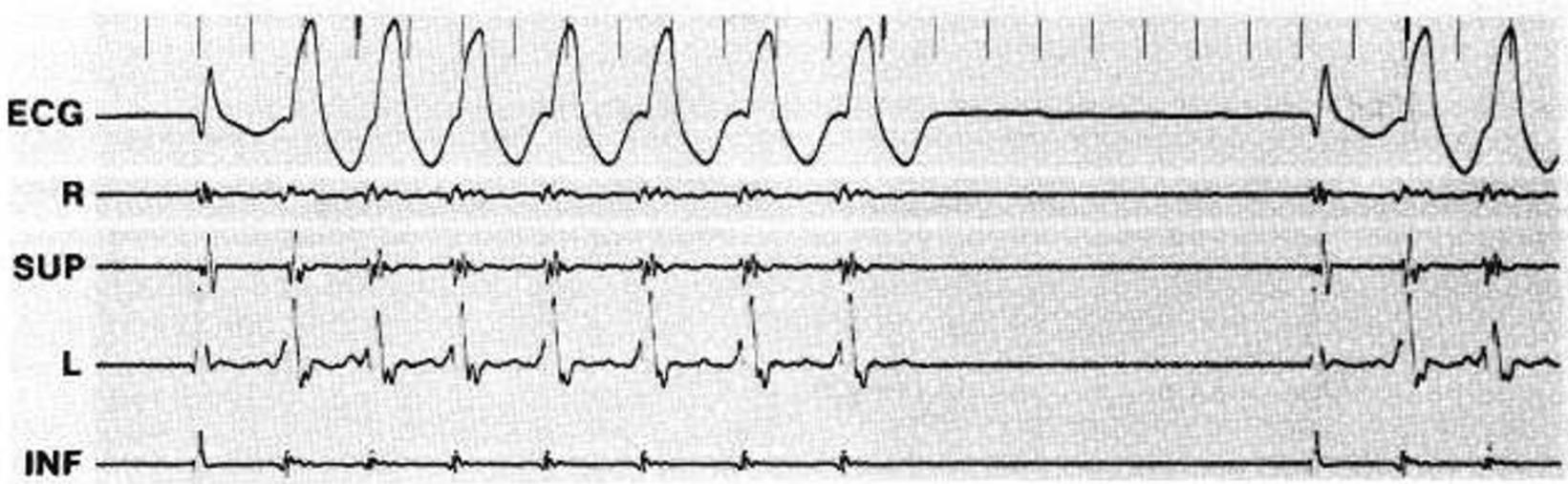

Fig. 7. Response to verapamil. Some ventricular tachycardia as in Figure 6. After verapamil, note the spontaneous termination and slower cycle length compared to Figure 6. All right ventricular electrograms occur before the onset of the QRS and occur within 20 ms of each other.

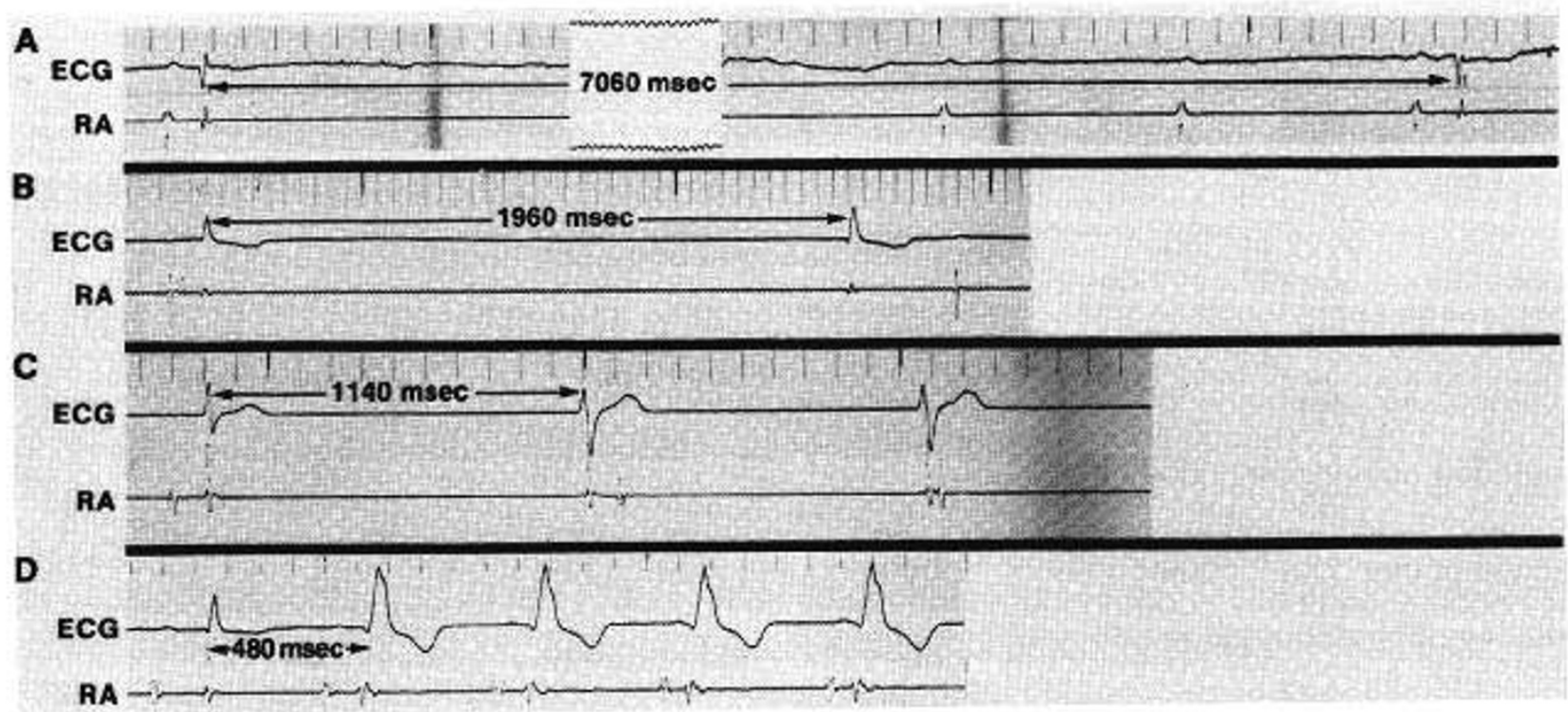

Fig. 8. Response to vagal stimulation. The beat on the far left of each tracing is the last sinus beat. $A$, control animal with a $7060-\mathrm{ms}$ pause and a sinus escape beat. There is atrioventricular block until the third atrial impulse (see right atrial electrogram) is conducted. $B$, right ventricular hypertension animal with junctional escape beat. $C$, ventriculotomy and right ventricular hypertension animal with ventricular escape beat. $D$, ventriculotomy and right ventricular hypertension animal with accelerated ventricular escape rhythm.

temic arterial pressure in all animals. The hypertrophic response in terms of wall thickness was approximately twice that produced by chronic right ventricular volume overload in dogs (3). In our model, not only did the right ventricular systolic pressure and wall thickness increase, but also the right ventricular end diastolic pressure and chamber size increased. Therefore, this model actually produced both right ventricular hypertrophy and dilation.

Electrophysiologically, the QRS duration prolonged in five of the six animals with right ventricular hypertension. This could be caused either by stretch of the right bundle branch, or diffuse slowing in conduction due to the stretch on the ventricular muscle, or the increased muscle mass requiring a longer time for depolarization. Since there was prolonged conduction from the right ventricular apex to base in these animals, the latter two explanations seem more likely.

These animals had both spontaneous and induced dysrhythmias. Other investigators have found dysrhythmias associated with ventricular pressure overload. In a heart-lung preparation, Vick (33) found an accelerated ventricular escape rate in dogs subjected to an increased afterload. Either ischemia associated with an elevated end diastolic pressure or stretch from the increased afterload could cause an increased rate of rise of phase 4 of the action potential, partial depolarization of cells, or a prolonged action potential duration, any one of which is dysrhythmogenic (27). Recently, Aronson (1) found ventricular dysrhythmias in rats with chronic left ventricular pressure overload.

In this model, the dogs had both right ventricular hypertrophy and right ventricular dilation. This group of animals is, therefore, more a model of right ventricular failure than unoperated pulmonary stenosis $(12,16)$.

Ventriculotomy and Right Ventricular Hypertension Group. The findings in this group were similar to those with right ventricular hypertension alone with two exceptions. First, the ventriculotomy and right ventricular hypertension group had the most prolonged intraventricular conduction and QRS duration in the acute period. This became similar to the right ventricular hypertension group in the chronic period. It appears that, in the acute period, the ventriculotomy and right ventricular hypertension were additive in prolonging conduction. The ventriculotomy and right ventricular hypertension group also had a greater prevalence of inducible ventricular dysrhythmias and the only spontaneous or inducible sustained ventricular tachycardia in the chronic period.

Although the majority of these animals had both prolonged intraventricular conduction and ventricular dysrhythmias, the observation of animals without prolonged conduction and with dysrhythmias implies that one is not always necessary for the other. Therefore, we hypothesize that both are separate byproducts of the abnormal cellular physiology created by the right 
ventricular hypertension and ventriculotomy, rather than a sequence of alteration leading to prolonged conduction which causes dysrhythmias. This observation, coupled to the data that the animals with dysrhythmias all had normal effective refractory periods of the ventricle, implies that the presence of dysrhythmias must be related to changes which cannot be measured in the whole ventricle but rather must be examined at the cellular level.

\section{MECHANISMS OF DYSRHYTHMIAS}

In both groups of animals with right ventricular hypertension, spontaneous an induced dysrhythmias were observed. Certain inferences about the mechanism can be made on the basis of the characteristics of these dysrhythmias. Recently several reviews have been published enumerating ways to distinguish between reentry, enhanced automaticity, and triggered activity in the clinical electrophysiology laboratory $(5,18,24,29,32)$.

In the animals with spontaneous dysrhythmias, the observation of fixed coupled premature ventricular contractions is not helpful in distinguishing a mechanism; however, the observation that, with shortening of the preceding cycle length, the coupling interval to the premature beat also shortens, implies the presence of triggered activity. This relationship of coupling interval is at present the only way without microelectrode studies to infer the presence of triggered activity $(25,30,36)$. Finally, the characteristic shortening of cycle lengths at the onset of accelerated ventricular rhythm or ventricular tachycardia implies either enhanced automaticity or triggered activity.

In the animals with dysrhythmias during electrophysiology study, the observation of inducible non-bundle branch reentry beats as well as inducible ventricular tachycardia implies either reentry or triggered activity $(4,23,28)$. Since in this model all of the right ventricular electrograms occurred early in the QRS complex of the induced dysrhythmias, it seems likely that the dysrhythmias did not reenter around the scar as a macro-reentry circuit, although this possibility cannot be completely ruled out on the basis of these data. While the induction of single nonbundle branch reentry beats in adults is relatively nonspecific, our data in children suggest that the induction of even a single non-bundle branch reentry beat is abnormal (15). The finding of accelerated ventricular escape in response to vagal stimulation implies enhanced automaticity; however, the induction of an accelerated ventricular rhythm with a similar morphology to the accelerated ventricular escape beat could imply triggered activity. In four animals, the induced dysrhythmias had characteristics suggesting triggered activity $(18,25,30,36)$ : they began in late diastole, there was a direct relationship between the inducing cycle length and the coupling interval to the induced beat; in one dog with induced ventricular tachycardia, the tachycardia rate was more rapid if the cycle length at the inducing stimulus was shorter.

\section{CONCLUSIONS}

In the dog with an otherwise normal right ventricle, ventriculotomy has no lasting dysrhythmogenic effect. In this model, chronic right ventricular hypertrophy and dilation are associated with prolonged intraventricular conduction and spontaneous and induced dysrhythmias. Ventriculotomy and right ventricular hypertension with dilation may be additive in prolongation of conduction and production of dysrhythmias. There was no association between properties of the whole ventricle (e.g. conduction time and refractory period) and dysrhythmias. Thus, the mechanism of dysrhythmias must be further evaluated at the cellular level. The mechanism for dysrhythmias may involve triggered activity.

\section{REFERENCES}

1. Aronson RS 1981 Afterpotentials and triggered activity in hypertrophied myocardium from rats with renal hypertension. Circ Res 48:720

2. Bassett AL, Gelband H 1971 Electrical and mechanical properties of cardiac muscle during chronic right ventricular volume overload. Rec Adv Stud Card Struct Metab 4:3

3. Brown FK, Brown WJ, Ellison RG, Hamilton WF 1968 Electrocardiographic changes during development of right ventricular hypertrophy in the dog. Am J Cardiol 21:223

4. Chua W, Singer D, Ten Eick R, Moran J 1982 Oscillatory activity in diseased human ventricular myocardium. Am J Cardiol 49:970

5. Cranefield PF 1977 Action potentials, afterpotentials and arrhythmias. Circ Res $41: 415$

6. Davidson RM 1976 Chronic accelerated ventricular rhythm. J Electrocardiol 9:249

7. Deanfield JE, McKenna WJ, Hallidie-Smith KA 1980 Detection of late arrhythmia and conduction disturbance after correlation of tetralogy of Fallot. Br Heart J 44:248

8. Fixler DE, Wheeler JM 1980 Dopamine infusion following ventriculotomy in dogs with right ventricular hypertrophy. Pediatr Res 13:343

9. Fuster V, McGoon DC, Kennedy MA: Long-term evaluation (12 to 22 years) of open heart surgery for tetralogy of Fallot. Am J Cardiol 46:635

10. Fuster V, McGoon M, Beahrs MM, Ritter DG, McGoon DC 1982 Long-term evaluation (11-24 years) of open heart surgery for pulmonary atresia with ventricular septal defect. Am J Cardiol 49:1035

11. Garson A, Nihill MR, McNamara DG, et al 1979 Status of the adult and adolescent following repair of tetralogy of Fallot. Circulation 59:1232

12. Garson A, McNamara DG, Cooley DA 1979 Tetralogy of Fallot in adults. In: Robert WC (ed) Congenital Heart Disease in Adults, Cardiovascular Clinics (Vol 10). FA Davis Co, Philadelphia, pp 341-364

13. Garson A 1981 Evaluation and treatment of chronic ventricular dysrhythmias in the young. Cardiovasc Rev Rep 2:1164

14. Garson A 1981 Ventricular dysrhythmias. In: Gillette PC, Garson A (eds) Pediatric Cardiac Dysrhythmias. Grune \& Stratton, New York, pp 295-360

15. Garson A, Porter CJ, Gillette PC, McNamara DG 1983 Ventricular tachycardia induction during electrophysiology study after repair of tetralogy of Fallot, J Am Coll Cardiol 1:1493

16. Garson A 1983 The Electrocardiogram in Infants and Children: A Systematic Approach. Lea \& Febiger, Philadelphia, p 214

17. Gelband H, Waldo AL, Kaiser GA, et al 1971 Etiology of right bundle branch block in patients undergoing total correction of tetralogy of Fallot. Circulation 44:1022

18. Gillette PC, Garson A 1981 Intracardiac electrophysiologic studies: use in determining site and mechnisms of dysrhythmias. In: Gillette PC, Garson A (eds) Pediatric Cardiac Dysrhythmias. Grune \& Stratton, New York, pp 77120

19. Harris AS, Oshima H, Boggs PB 1966 Changes in infarction arrhythmias resulting from changes in arterial pressure. Am J Physiol 210:526

20. Josephson ME, Horowitz LN, Farshidi A, Kastor JA 1978 Recurrent sustained tachycardia. I. Mechanisms. Circulation 57:431

21. Kavey REW, Byrum C, Blackman M, Sondheimer H 1984 On the etiology of ventricular arrhythmias after repair of tetralogy of Fallot. Clin Res, in press

22. Marcus ML, Eckburg DL, Braxmeier JL, et al 1977 Effects of intermittent pressure loading on the development of ventricular hypertrophy in the cat Circ Res 40:484

23. Mary-Rabine L, Hordof AJ, Danilo P, Malm JR, Rosen MR 1980 Mechanisms for impulse initiation in isolated human atrial fibers. Circ Res 47:267

24. Mason JW 1982 Efficacy of verapamil in recurrent ventricular tachycardia. Am J Cardiol 49:1015

25. Moak J, Rosen MR 1982 The effects of pacing on ouabain-induced sustained rhythmic activity. Circulation 66 (suppl II):79

26. Myerburg RJ, Gelband H, Nilsson K, et al 1977 Long-term electrophysiological abnormalities resulting from experimental myocardial infarction in cats. Circ Res 41:73

27. Penefsky ZJ, Hoffman BF 1963 Effects of stretch on mechanical and electrical properties of cardiac muscle. Am J Physiol 204:433

28. Rosen MR, Gelband H, Merker C, Hoffman BF 1973 Mechanisms of digitalis toxicity: effects of ouabain on phase four of canine Purkinje fibers transmembrane potentials. Circulation 47:681

29. Rosen MR, Danilo P 1980 Effect of tetrodotoxin, lidocaine, verapamil and AHR-2666 on ouabain-induced delayed afterdepolarization in canine Purkinje fibers. Circ Res 46:117

30. Rosen MR, Fisch C, Hoffman BF, Danilo P, Lovelace DE, Knoebel SR 1980 Can accelerated atrioventricular escape rhythms be explained by delayed afterdepolarizations? Am J Cardiol 45:1272

31. Rosen MR, Reder RF 1981 Does triggered activity have a role in the genesis of cardiac arrhythmias? Ann Intern Med 94:794

32. Sung RJ, Shen EN, Shapiro W, Morady F, Davis J 1982 Verapamil and ventricular tachycardias caused by reentry, abnormal automaticity and triggered activity. Circulation 66 (suppl II):271

33. Vick RL 1963 Effects of increased transmural pressures upon atrial and ventricular rhythms in the dog heart-lung preparation. Circ Res 13:39

34. Wallenstein S, Zucker C, Fleiss JL 1980 Some statistical methods useful in circulation research. Circ Res 47:1

35. Webeau G 1953 L'age du chien et cellui de l'homme; essai de statistique sur la mortalite canine. Bull Acad Vet 26:229

36. Wellens HJJ, Brugata $P$, Vanagt E, Ross DL, Bar FW 1981 New studies in triggered activity. In: Harrison D (ed) Cardiac Arrhythmias: a Decade of Progress. GK Hall, Boston, pp 601-610

37. Wolff GS, Rowland TW, Ellison RC 1972 Surgically induced right bundle branch block with left anterior hemiblock: an ominous sign in postoperative tetralogy of Fallot. Circulation 46:587 